\title{
1 Liposomes Under Real Cell Conditions Behave Like Real Cells with a Single Pulsed
}

\section{$2 \quad$ Electric Field}

3

4 Gen Urabe, ${ }^{1 *}$ Masaharu Shimada, ${ }^{1}$ Takumi Ogata, ${ }^{1}$ Sunao Katsuki ${ }^{2}$

5

61 Room 306, Kurokami South W2, Graduate School of Science and Technology,

7 Kumamoto University, Kurokami 2-39-1 Chu-o-ku, Kumamoto City, Kumamoto,

8 860-8555, Japan

92 Room 306, Kurokami South W2, Institute of Pulsed Power Science, Kumamoto

10 University, Kurokami 2-39-1 Chu-o-ku, Kumamoto City, Kumamoto 860-8555, Japan

\section{${ }^{*}$ Corresponding author}

13 Email: g.urabe@st.cs.kumamoto-u.ac.jp

\section{Abstract}

16 Liposomes are widely assumed to present a straightforward physical model of cells.

17 However, almost all previous liposome experiments with pulsed electric fields (PEFs)

18 have been conducted in low-conductivity liquids, a condition that differs significantly 
19 from that of cells in medium. Here, we prepared liposomes consisting of soy bean

20 lecithin and cholesterol, at a molar ratio of 1:1, in higher-conductivity liquid that

21 approximated the conditions of red blood cells in phosphate-buffered saline, with inner

\section{Keywords}

26 Liposome; Vesicle; Pulsed electric field; Conductivity; Real cell condition; Lipid composition

\section{Introduction}

30 Bioelectrics, which studies the relationship between electricity and biology, has been

31 the subject of both basic and applied research of note [1-9]. Electropores induced on plasma membranes may trigger responses in cells and tissues to pulsed electric fields studies have managed to completely mimic the phenomenon in cells. In the case of cells, 
37 subsequently, actin patches appear on the surface, after which plasmid DNA is taken

38 into the cells [3,21]. In a study using liposomes, plasmid DNA traversed the lipid

39 membrane without accumulating [22]. Rols et al. assumed actin as the source of the

40 difference and suggested conducting a similar experiment using actin-containing

41 liposomes.

42 Little dye was seen in liposomes without actin, but liposomes with actin proceeded

43 taking dye more than $146 \mathrm{~s}$, and the latter reaction was similar to those of cells under a

44 PEF [23]. However, those liposomes were prepared in liquid with much lower

45 conductivity than is seen in cytoplasm and extracellular liquid (phosphate-buffered

46 saline [PBS]) [24-27]. Because liquid conductivity influences substance influx through

47 electropores on cells [28-30] and low-conductivity conditions differ from those of cell

experiments, experiments that approximate real cytoplasm and media will require

adjustment of the conditions. Furthermore, although the liposomes exhibited accurate

lipid molar fractions, it is unlikely that their composition was similar to that of real cell

membranes. As membrane composition can influence reactions to PEFs, liposome

compositions that are closer to those of a real plasma membrane are necessary. To that 
55 inner- and outer-liquid conductivities comparable to those of red blood cells $(0.6 \mathrm{~S} / \mathrm{m})$

56 and PBS (1.6 S/m) and whose membranes were composed of soybean lecithin and

57 cholesterol. We adjusted the conductivity of liposomes' inner liquid to that of red blood

cells because their value had already been reported, and most previous simulation

studies preferred this value [31]. Because soybean lecithin is not a purified lipid, but an

extract, we assumed that the composition of soybean lecithin was close to that of real

Materials

For liposome preparation, we purchased soy bean lecithin, cholesterol, liquid paraffin, phosphocholine (POPC), chloroform, glucose, and sucrose from Wako and Texas Red- 
osmotic pressure of PBS(-) was $306 \mathrm{mOsm} / \mathrm{L}$. The osmotic pressure of the $308 \mathrm{mM}$

74 glucose solution was theoretically $308 \mathrm{mOsm} / \mathrm{L}$. Conductivities of the inner and outer

75 liquids were 0.64 and $1.64 \mathrm{~S} / \mathrm{m}$, respectively. Cholesterol, DOPC, and POPC were stored at a temperature of $-20^{\circ} \mathrm{C}$.

\section{Liposome preparation}

\section{Lecithin liposome}

81 Liposome production followed the water-in-oil emulsion method [32]. We estimated the

soy bean lecithin molar weight at 758.06 and then dissolved $0.53 \mathrm{mg}$ of soy lecithin in

$500 \mu \mathrm{L}$ of liquid paraffin at $80^{\circ} \mathrm{C}$ by vortex $(1.4 \mathrm{mM})$. After storing the sample at a

temperature of $80^{\circ} \mathrm{C}$ for 10 min with the cap of the microtube left open, we added $50 \mu \mathrm{L}$

of the inner liquid and vortexed it for $10 \mathrm{~s}$ and then immediately put the sample on ice

for $10 \mathrm{~min}$ to stabilize the emulsions. Next, $400 \mu \mathrm{L}$ of the emulsion solution was put on

$400 \mu \mathrm{L}$ of the outer liquid, which had been on ice. The volume ratio of the emulsion

solution and the outer liquid was 400:400. After treating the sample under 
91 liposomes.

93 Lecithin-cholesterol liposomes and fluorescent liposomes

94 To produce cholesterol-containing lecithin liposomes (at a molar ratio of

95 lecithin/cholesterol $=1: 1)$, we added $4 \mu \mathrm{L}$ of cholesterol solution to a heated liquid

96 paraffin-lecithin solution at a temperature of $80^{\circ} \mathrm{C}$ and then mixed them by vortex to

97 adjust the cholesterol concentration to $1.4 \mathrm{mM}$. For a lecithin/cholesterol molar ratio of

$98 \quad 1: 1.5$ and 2:1 liposomes, the amounts of added cholesterol solution were 6 and $2 \mu \mathrm{L}$. For

99 fluorescent labeling, $2 \mu \mathrm{L}$ of Tex Red-DHPE was added simultaneously to make a final

100 concentration of $3.6 \mu \mathrm{M}$ (with a molar ratio of $0.13 \mathrm{~mol} \%$ ). The sample was stored at a

101 temperature of $80^{\circ} \mathrm{C}$ for $10 \mathrm{~min}$ with the cap of the microtube left open, and the rest of

102 the protocol was the same as that of lecithin liposome.

104 DOPC liposomes and POPC liposomes

105 To produce DOPC liposomes, DOPC and cholesterol liquids were mixed in $500 \mu \mathrm{L}$ of

106 liquid paraffin at the following ratios: 4:0 for DOPC-only liposomes, 4:4 for

107 DOPC/cholesterol $=1: 1($ molar ratio $), 2: 4$ for DOPC/cholesterol $=1: 2($ molar ratio $)$, and

$1082: 6$ for $\mathrm{DOPC} /$ cholesterol $=1: 3$ (molar ratio). In the case of POPC, the POPC and 
109 cholesterol liquids were mixed at the following ratios; 4:0 for POPC-only liposomes and

$1104: 4$ for POPC/cholesterol = 1:1 (molar ratio). The sample was stored at a temperature of

$11180^{\circ} \mathrm{C}$ for $10 \mathrm{~min}$ with the cap of the microtube left open, and the rest of the protocol was

112 the same as that of lecithin liposome

\section{Calcium-ion influx detection}

115 The same osmotic pressure between inner and outer liquid

116 First, $50 \mu \mathrm{g}$ of Fluo-8(R) sodium salt (Cosmo Bio) was dissolved in $62.5 \mu \mathrm{L}$ of Milli-Q,

117 and $1 \mu \mathrm{L}$ of the solution was diluted in $50 \mu \mathrm{L}$ of the inner liquid for a final Fluo-8

118 concentration of $20 \mu \mathrm{M}$. For the calcium-ion flow experiment, liposomes were prepared

119 with the Fluo-8-containing inner liquid. After preparing the liposome sample, a solution

120 of D-PBS(+) preparation with a $\mathrm{Ca}$ and $\mathrm{Mg}$ reagent solution $100 \times$ (Nacalai Tesque) was

121 added at $1 \%(\mathrm{v} / \mathrm{v})$ to mix the calcium ions in the outer liquid.

\section{Different osmotic pressures between inner and outer liquid}

124 To strengthen calcium influx, we set the outer-liquid osmotic pressure at half of that of

125 the inner liquid. The inner-liquid composition was the same as that used to text Ca-ion

126 influx, but in the case of the outer liquid, the PBS (-) was diluted twice with Milli-Q. The 
127 rest of the protocol was the same as that of no osmotic pressure difference.

\section{PEF application system and microscopy}

130 We used two slices of a platinum plate, which are $0.2-\mathrm{mm}$ thick and $2.7-\mathrm{mm}$ wide, as

131 electrodes. The electrodes were fixed with a $640-\mu \mathrm{m}$ gap on a glass slide and connected

132 to the PEF generator. We set the glass slide-electrode device on a fluorescent

133 microscope (Leica, DMi8) combined with a digital camera (Canon, EOS 8000D). Movies

134 were recorded at 67 frames per second. The details of microscopy are provided in a

135 previous paper [33].

\section{$137 \quad$ Results}

138 We referred to HeLa cells, which were familiar to us, for the lipid molar ratio of cell

139 membranes, facilitating a comparison of liposome and cell results. As PC and

140 cholesterol constitute primarily of HeLa cell membranes, their molar ratio was 1:1, and

141 other lipids represented only minor parts [34,35], we mixed lecithin and cholesterol at a

142 molar proportion of $1: 1$. 
145 When we applied a single $1.1 \mathrm{kV} / \mathrm{cm}, 400 \mu \mathrm{s}$ PEF to the liposomes, the liposomes

146 divided spontaneously (Fig. 1A, B, and C). To determine the optimal PEF condition to

147 induce division, we scanned the pulse duration and electric field intensity, showing that

148 a 400 or $500 \mu \mathrm{s}, 1.1 \mathrm{kV} / \mathrm{cm}$ PEF appeared to have the potential to induce division (Table.

149 1). A PEF with identical energy but a shorter pulse did not promote self-division (Table.

150 2). Because a single $1.1 \mathrm{kV} / \mathrm{cm}, 400 \mu \mathrm{s}$ PEF can theoretically increase the PBS

151 temperature by $2^{\circ} \mathrm{C}$, the thermal influence may be small. These results suggest that

energy was not an important factor in the division.

154 Here Fig. 1.

155 Here Table. 1

156 Here Table. 2

Do cholesterol and lecithin have division ability?

159 As liposomes without cholesterol did not divide, it is possible that cholesterol has the 
167 To evaluating which elements contributed to the liposome division, we prepared the

168 liposomes, with each lipid and cholesterol at a 1:1 molar proportion. DOPC produced

169 lecithin-like liposomes, but POPC did not (Fig. 3). Because POPC had higher phase

171 [36], we anticipated that the surface aggregation on POPC liposomes may be aggregates

172 of gel-phase POPC. We did not prepare DPPC liposomes because DPPC has a much

173 higher phase transition temperature, making it unlikely for DPPC liposomes to produce

174 lecithin-like liposomes. No division occurred in DOPC liposomes, which most likely division. 


\section{Conductivity of liquids related to the division}

183 When we lowered the conductivity of both inner and outer liquids to $105 \mu \mathrm{S} / \mathrm{m}$, no

184 division occurred (lecithin/cholesterol = 1:1) (Table. 3). Because the emulsion method

185 required heavier inner liquids than outer liquids, we mixed glucose solution and PBS (-)

186 for the inner liquid to ensure that inner conductivity decreased to $0.6 \mathrm{~S} / \mathrm{m}$. For the same

187 reason, we did not execute the experiments with $1.6 \mathrm{~S} / \mathrm{m}$ inner liquid. Instead, $0.6 \mathrm{~S} / \mathrm{m}$

188 for the inner liquid and $1.6 \mathrm{~S} / \mathrm{m}$ for the outer liquid (the same as for the red blood cells in

189 PBS) were deemed appropriate.

$191 \quad$ Here Table. 3.

\section{Ca-ion influx had little relation to division}

194 To analyze ion influx under the PEF that induced the liposome division, we attempted

195 to observe Ca-ion influx into liposomes. However, we detected no Ca-ion flow into

196 liposomes after a single PEF (Fig. 4). Lecithin liposomes, the surfaces of which are not

197 smooth, collapsed immediately after PEF exposure (Supplementary Fig. 1A). When the

198 liposomes disintegrated, green fluorescence emerged from the outside of the liposomes, 
199 indicating that Fluo-8 in the liposomes had seeped to the surroundings and connected to

200 Ca ions (Supplementary Fig. 1B). This proved that our method could detect Ca-ion

201 influx. Because a Ca ion is much smaller than dyes such as propidium iodide, Ca ions

202 are prone to flowing across lipid membranes faster than dyes [37]. Our failure to detect

203 a Ca influx indicated that the extent of liquid flow accompanied by the single $1.1 \mathrm{kV} / \mathrm{cm}$,

$204400 \mu \mathrm{s}$ PEF was small.

205

206 Here Fig. 4.

208 Endocytosis-like phenomena are also induced

209 Lecithin-cholesterol liposomes sometimes showed that lipid patches were detached from

210 the membrane surface as in endocytosis (Fig. 5A). Additionally, lipids aggregated on the

211 membrane just after PEF application (Fig. 5B). However, on DOPC-cholesterol

212 liposomes, which did not have division ability, lipid aggregation and patch uptake did

213 not occur (Fig. 5 C, D).

215 Here Fig. 5. 


\section{Discussion}

\section{Why did liposomes divide?}

219 Why current conditions trigger liposome division and endocytosis-like behavior

220 remains unknown. Previous studies have reported that electrofusion of liposomes

221 promotes spontaneous liposome division, explaining the phenomenon in terms of

222 thermodynamics; as the amount of lipid per liposome increased by electrofusion, the

223 system became unstable, and consequently, liposomes divided to increase entropy and

224 stabilize the system [38,39]. In our case, however, electrofusion did not precede division,

225 so the previous theory did not explain our results. Because liposome division

226 accompanied liposome deformation, it seemed possible to describe the phenomenon in

227 terms of liposome volume change, but the influence of volume change is likely small

228 because the osmotic pressures of the inner and outer liquids were almost identical. In

229 fact, calcium-ion influx was too small to detect.

230 In addition, we have no reasonable explanation on why liposome divisions did not

231 occur in previous studies. However, our study with less-conductive inner and outer

232 liquids suggests that liquid conductivity is one of the determining factors. Indeed, one

233 paper using buffered solution as an electroformation buffer showed vesicle budding

234 without releasing daughter vesicle [40]. 


\section{Membrane conditions may be related to liposome division}

237 We increased Ca-ion influx by reducing the osmotic pressure of the outer liquid

238 compared with the inner liquid. Using $154 \mathrm{mM}$ glucose solution with a conductivity of

$2390.7 \mathrm{~S} / \mathrm{m}$ and a theoretical osmotic pressure of $154 \mathrm{mOsm} / \mathrm{L}$ as the outer liquid and

240 PBS(-)-glucose solution with a conductivity of $0.6 \mathrm{~S} / \mathrm{m}$ and a theoretical osmotic

241 pressure of $308 \mathrm{mOsm} / \mathrm{L}$ as the inner liquid, Ca-ion influx was not detected in lecithin

242 liposomes (Supplementary Fig. 2A and B), whereas lecithin-cholesterol liposomes

243 disintegrated with Ca-ion influx (Supplementary Fig. 2C and D). These tendencies

244 coincided with those of previous studies that revealed that liposomes, including charged

245 lipids, such as cholesterol and POPG, were likely to collapse more easily due to PEF

246 exposure compared with those without charged lipids [41,42]. This prompted the

247 question of whether charged lipids can trigger liposome division. We therefore examined

248 the behavior of lecithin-POPG liposomes and lecithin-POPC liposomes. POPG has a

249 negative charge, whereas POPC does not, but both have similar hydrophobic backbones

250 with one saturated and one unsaturated fatty acid chain. Both POPG-containing

251 liposomes and POPC-containing liposomes divided (Supplementary Fig. 2E and F). This

252 suggested that it is not lipid charge but membrane condition that induces liposome 
253 division. In fact, Riske et al. reported changes in lipid composition in a plasma

254 membrane during mitosis [43]. Determining which type of lipid can induce liposome

255 division with lecithin should be subjected to a screening wand, and we intend to follow

256 this line of inquiry in the future.

\section{$258 \quad$ Future tasks}

259 There are several important points to examine in the future: the relationship between

260 PEF conditions and the time lag from PEF application to division or endocytosis, the

261 influence of inner-liquid viscosity, the relationship between PEF conditions and the size

262 of mother liposomes and that of daughter liposomes, PEF direction and division or

263 endocytosis polarities, and how membrane composition or conditions affect the division.

\section{Acknowledgments}

266 This study was partially supported by a Grant-in-Aid for Scientific Research

267 (17H03220). The authors would like to thank Enago (www.enago.jp) for the English

language review. 
271 G.U, M.S, and T.O contributed to the experiments. S.K supervised and managed this 272 project.

\section{$274 \quad$ References}

275 [1] D.L. Perrier, L. Rems, P.E. Boukany, Lipid vesicles in pulsed electric fields: Fundamental principles of the membrane response and its biomedical applications, Adv. Colloid Interface Sci. 249 (2017) 248-271. doi:10.1016/j.cis.2017.04.016.

[2] Z.A. Levine, P.T. Vernier, Life cycle of an electropore: Field-dependent and 27-36. doi:10.1007/s00232-010-9277-y. Electrotransfer: A Mechanistic Perspective, Curr. Gene Ther. 16 (2016) 98-129. doi:10.2174/1566523216666160331130040.

286 [5] K. Heileman, J. Daoud, M. Tabrizian, Dielectric spectroscopy as a viable biosensing 
289 [6] S. Sundelacruz, M. Levin, D.L. Kaplan, Role of membrane potential in the regulation doi:10.1007/s12015-009-9080-2.

[7] S.N. Iwasa, R. Babona-Pilipos, C.M. Morshead, Environmental Factors That

[10] M.P. Stewart, R. Langer, K.F. Jensen, Intracellular delivery by membrane doi:10.1021/acs.chemrev.7b00678. Chemotherapy induces tumor clearance independent of apoptosis, Cancer Res. 68 
[13] T.B. Napotnik, M. Reberšek, T. Kotnik, E. Lebrasseur, G. Cabodevila, D. Miklavčič,

311 [14] M. Leguèbe, A. Silve, L.M. Mir, C. Poignard, Conducting and permeable states of cell validated by the experiments, J. Theor. Biol. (2014). doi:10.1016/j.jtbi.2014.06.027.

314 [15] E.E. Vaughan, D.A. Dean, Intracellular trafficking of plasmids during transfection is mediated by microtubules, Mol. Ther. 13 (2006) 422-428. doi:10.1016/j.ymthe.2005.10.004. via the electropermeome measuring and modeling single nanosecond perturbations, Sci. Rep. (2017). doi:10.1038/s41598-017-00092-0. doi:10.1016/j.bioelechem.2017.08.007. 
[19] E. Karatekin, O. Sandre, H. Guitouni, N. Borghi, P.H. Puech, F. Brochard-Wyart, Cascades of transient pores in giant vesicles: Line tension and transport, Biophys. J. 84 (2003) 1734-1749. doi:10.1016/S0006-3495(03)74981-9. vesicles in electric fields, Soft Matter. 3 (2007) 817-827. doi:10.1039/b703580b.

[21] C. Rosazza, H. Deschout, A. Buntz, K. Braeckmans, M.P. Rols, A. Zumbusch, Endocytosis and Endosomal Trafficking of DNA After Gene Electrotransfer In Vitro, Mol. Ther. - Nucleic Acids. (2016). doi:10.1038/mtna.2015.59. negatively charged macromolecules, Soft Matter. 7 (2011) 3872-3881. doi:10.1039/c0sm01389g.

[23] D.L. Perrier, A. Vahid, V. Kathavi, L. Stam, L. Rems, Y. Mulla, A. Muralidharan, G.H. Koenderink, M.T. Kreutzer, P.E. Boukany, Response of an actin network in doi:10.1038/s41598-019-44613-5. 
characterization of the archaeal transporter NCX_Mj using solid supported doi:10.1085/jgp.201611587.

[25] E.M. Schmid, D.L. Richmond, D.A. Fletcher, Reconstitution of proteins on electroformed giant unilamellar vesicles, Elsevier Ltd, 2015. doi:10.1016/bs.mcb.2015.02.004.

349 [26] W. Li, Q. Wang, Z. Yang, W. Wang, Y. Cao, N. Hu, H. Luo, Y. Liao, J. Yang, Impacts doi:10.1016/j.colsurfb.2015.11.020. in a flow chamber, Biochim. Biophys. Acta - Biomembr. 1712 (2005) 152-160. exists only in certain electroporation buffers, PLoS One. 11 (2016). doi:10.1371/journal.pone.0159434. of mammalian cells by high-intensity, ultra-short pulses of submicrosecond duration, 
[30] J. Li, W. Tan, M. Yu, H. Lin, The effect of extracellular conductivity on electroporation-mediated molecular delivery, Biochim. Biophys. Acta - Biomembr. 1828 (2013) 461-470. doi:10.1016/j.bbamem.2012.08.014.

[31] A. Ivorra, Tissue Electroporation as a Bioelectric Phenomenon: Basic Concepts, in: 2010: pp. 23-61. doi:10.1007/978-3-642-05420-4_2.

[32] K. Nishimura, T. Hosoi, T. Sunami, T. Toyota, M. Fujinami, K. Oguma, T. Matsuura, by flow cytometry, Langmuir. 25 (2009) 10439-10443. doi:10.1021/la902237y.

Cells, IEEE Trans. Biomed. Eng. $66 \quad$ (2019) 2259-2268.

[34] D.B. Weinstein, J.B. Marsh, M.C. Glick, L. Warren, Membranes of animal cells. IV. http://www.jbc.org/content/244/15/4103. characterization of the plasma and smooth membranes of hela cells, Arch. Biochem. 
Biophys. 128 (1968) 51-69. doi:10.1016/0003-9861(68)90008-8.

[36] Q. Li, X. Wang, S. Ma, Y. Zhang, X. Han, Electroformation of giant unilamellar vesicles in saline solution, Colloids Surfaces B Biointerfaces. 147 (2016) 368-375. doi:10.1016/j.colsurfb.2016.08.018.

[37] B.L. Ibey, J.C. Ullery, O.N. Pakhomova, C.C. Roth, I. Semenov, H.T. Beier, M. pulses are less efficient at electropermeabilization and killing cells than monopolar pulses, Biochem. Biophys. Res. Commun. 443 (2014) 568-573. doi:10.1016/j.bbrc.2013.12.004.

[38] H. Shiomi, S. Tsuda, H. Suzuki, T. Yomo, Liposome-based liquid handling platform 5-10. doi:10.1371/journal.pone.0101820. and budding of giant phospholipid vesicles containing macromolecules, Proc. Natl. Acad. Sci. 109 (2012) 5942-5947. doi:10.1073/pnas.1120327109. subjected to electric pulses, Soft Matter. 5 (2009) 1983-1986. doi:10.1039/b900548j. 

doi:10.1039/b901963d. 


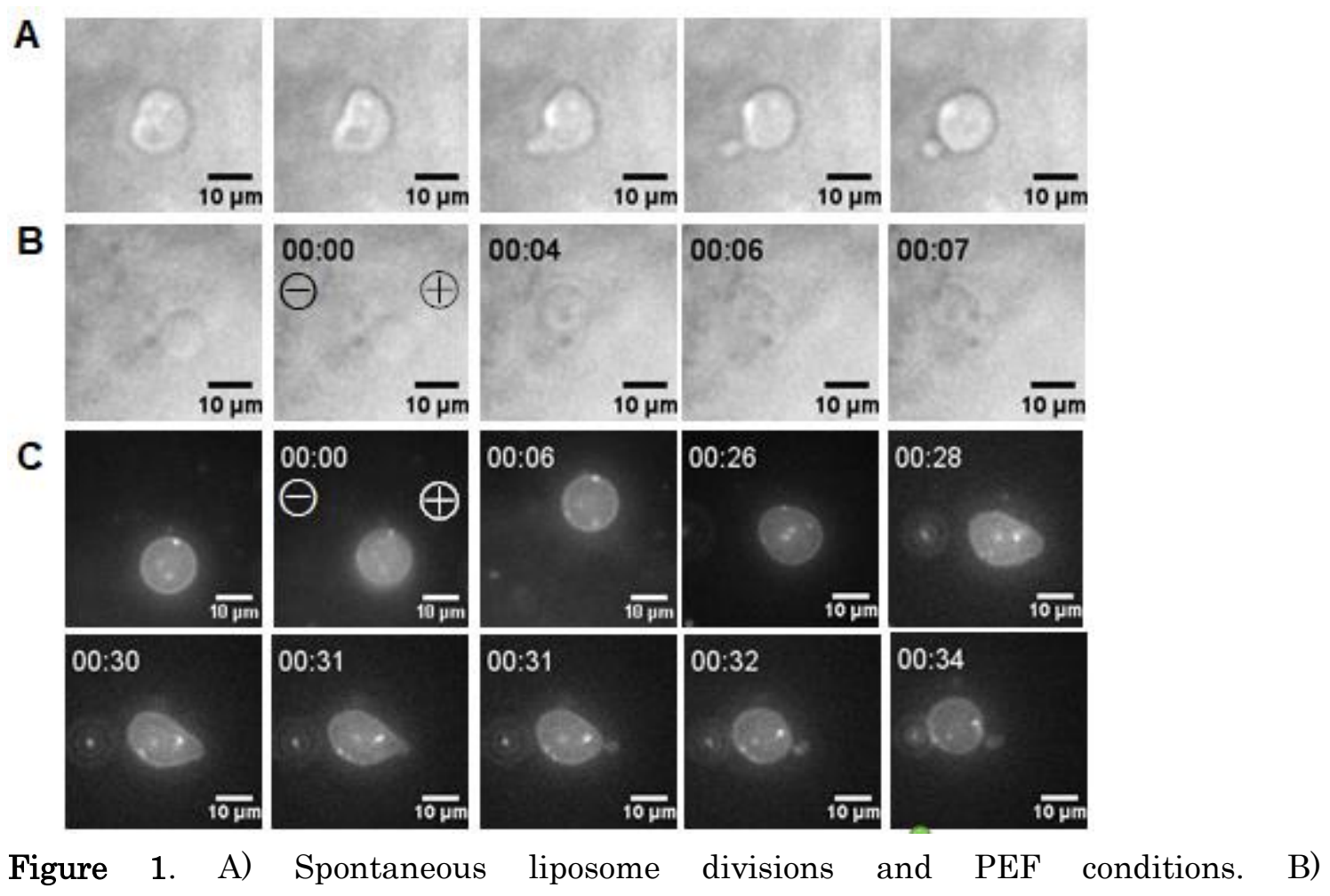

417 Figure 1. A) Spontaneous liposome divisions and PEF conditions. B)

418 Lecithin/cholesterol = 1:1 (molar ratio) liposomes divided spontaneously after a 1.1

$419 \mathrm{kV} / \mathrm{cm}, 400 \mu \mathrm{s}$ single PEF application. As we did not measure the time, there is no time

420 display in series A. All time displays are in seconds. "00:00" = timing of PEF application.

421 C. Liposomes with lecithin/cholesterol/texas red-DHPE $=1.4: 1.4: 0.0036 \mathrm{mM}$. $\oplus=$ the

422 anode side of the electrode; $\ominus=$ the cathode side of the electrode. 
bioRxiv preprint doi: https://doi.org/10.1101/860841; this version posted December 1, 2019. The copyright holder for this preprint (which

A
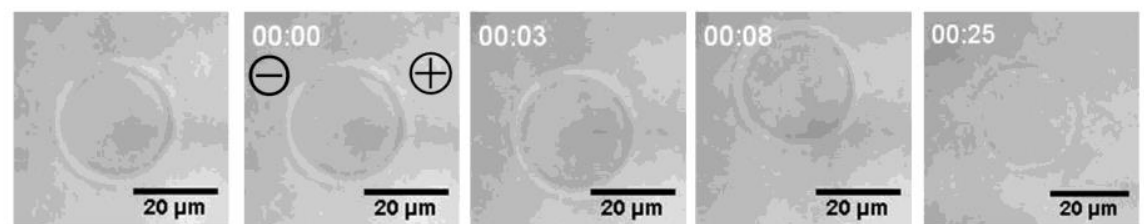

B
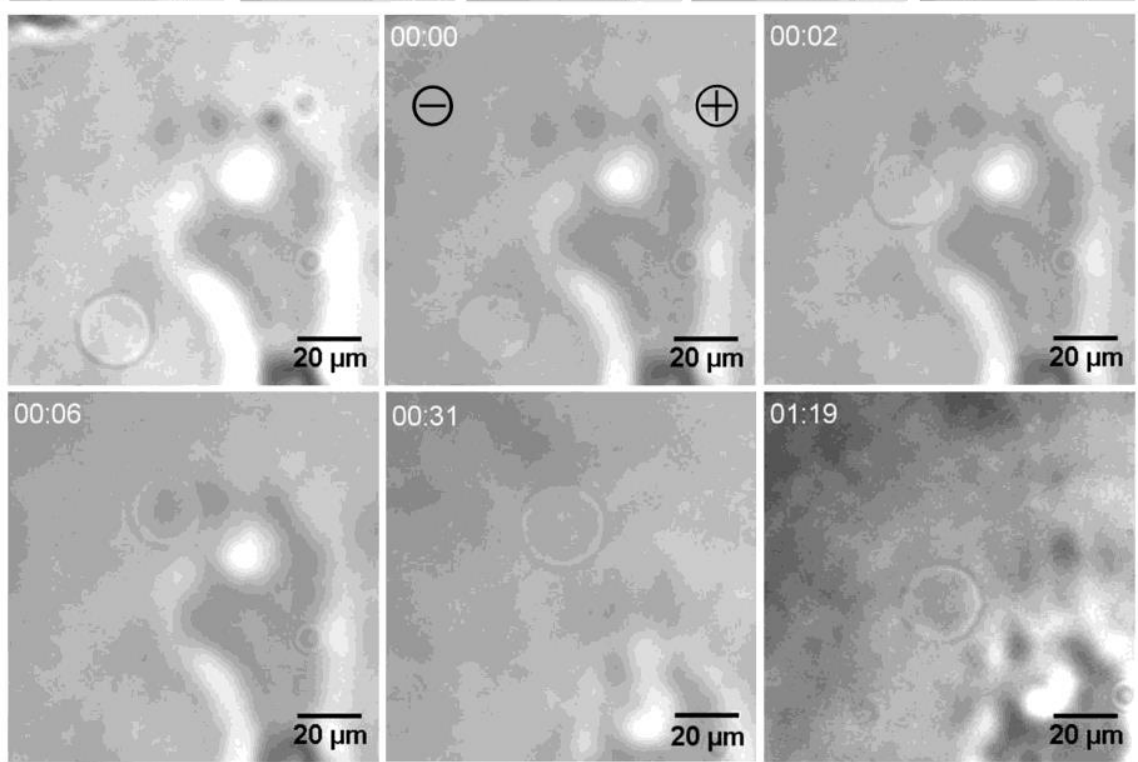

$\oplus$

C
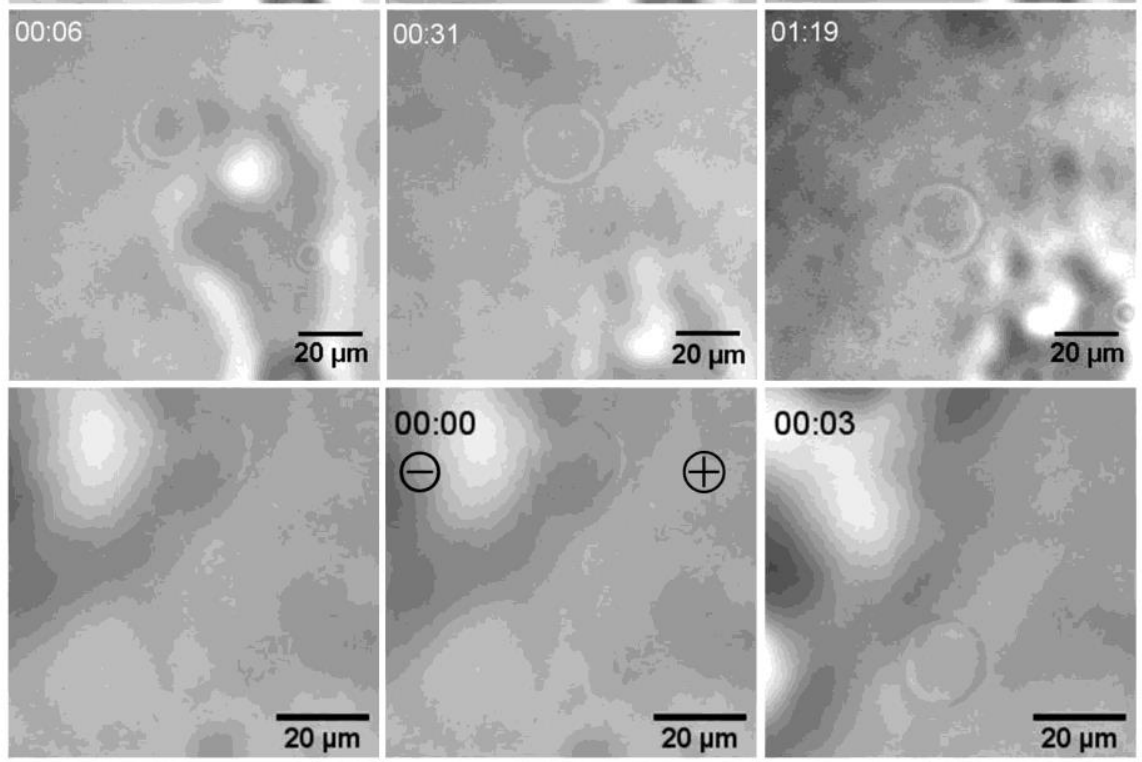

$\Theta$

$\oplus$

00:03

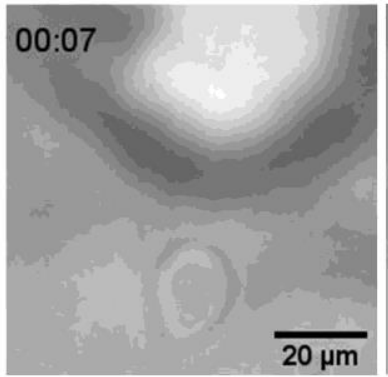

$\overline{20 \mu \mathrm{m}}$

$20 \mu \mathrm{m}$

D
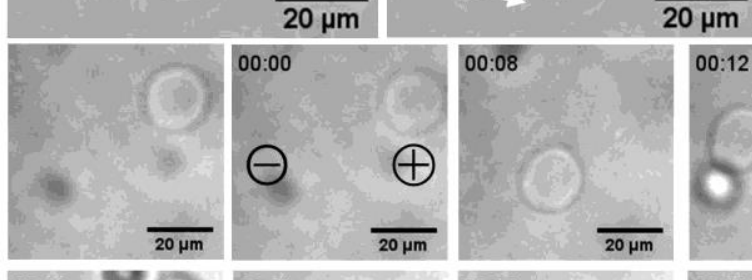

$20 \mu \mathrm{m}$
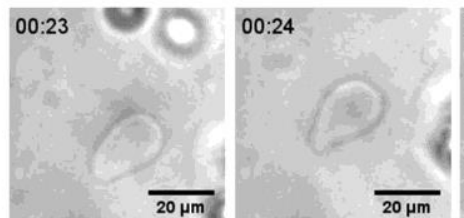

00:25

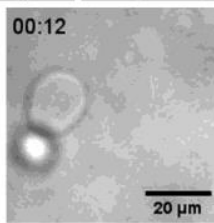

00:20

00:25

00:08

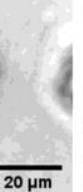

$20 \mu \mathrm{m}$

$00: 27$
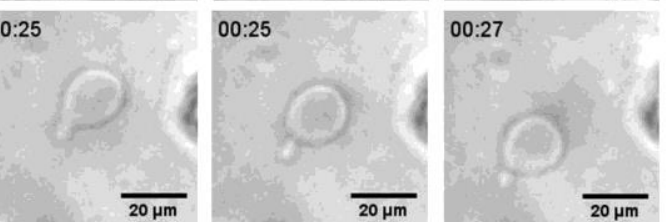
424 Figure 2. Reactions of several liposome types with $1.1 \mathrm{kV} / \mathrm{cm}, 400 \mu \mathrm{s}$ single PEF. A)

425 Lecithin-only liposomes did not divide. B) Lecithin/cholesterol $=2: 1$ (molar ratio)

426 liposomes did not divide. C) Lecithin/cholesterol = 1:1 (molar ratio) liposomes budded

427 daughter liposomes. The white arrow indicates the budding position. (D)

428 Lecithin/cholesterol = 1:1.5 (molar ratio) budded daughter liposomes. All time displays

429 are in seconds. "00:00" = timing of PEF application. $\oplus=$ the anode side of the electrode;

$430 \ominus=$ the cathode side of the electrode.

431

432

433

434

435

436

437

438

439

440

441

442

443

444

445

446

447

448

449

450 
A
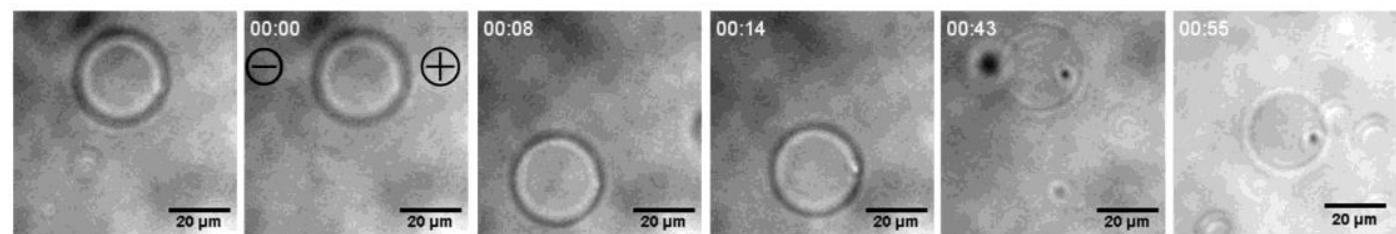

B
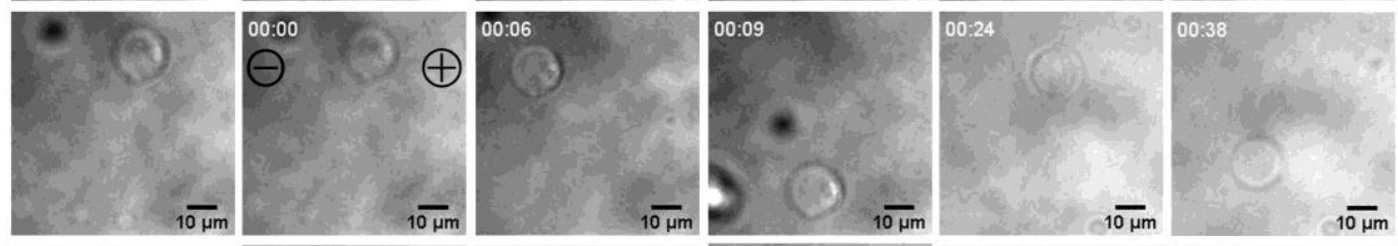

C
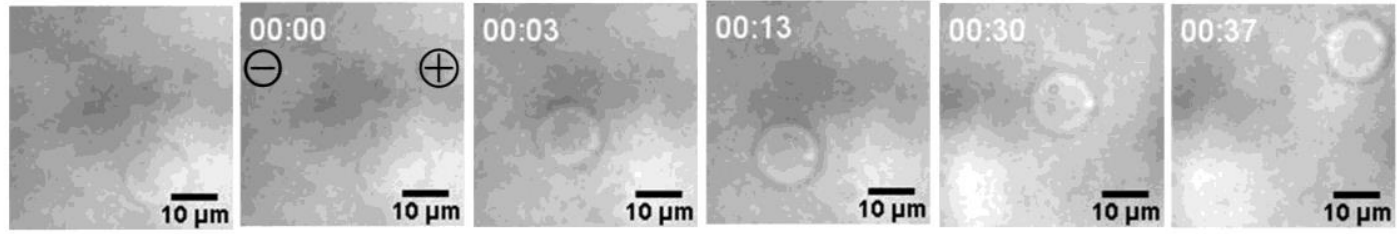

D

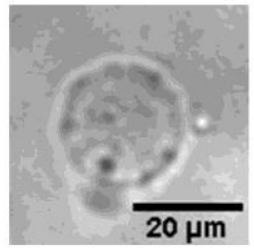

E

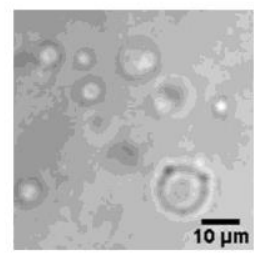

$\mathbf{F}$

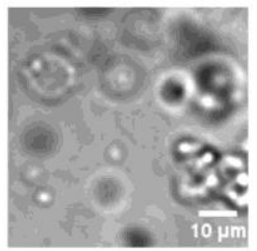

452

453

454

455

456

457

458

459

460

461

462

463

464

465

466

467

468

469

470

471

Figure 3. Liposomes without lecithin did not exhibit division. $\mathrm{PEF}$ was $1.1 \mathrm{kV} / \mathrm{cm}, 400$ $\mu$ s single pulse. A) DOPC/cholesterol = 1: 1 (molar ratio). B) DOPC/cholesterol =1:2 (molar ratio). C) DOPC/cholesterol = 1:3 (molar ratio). D) POPC-only liposome. $\mathrm{E}$ and F) $\mathrm{POPC} /$ cholesterol = 1:1 (molar ratio). All time displays are in seconds. "00:00" = timing of PEF application. $\oplus=$ the anode side of the electrode; $\ominus=$ the cathode side of the electrode. 
A

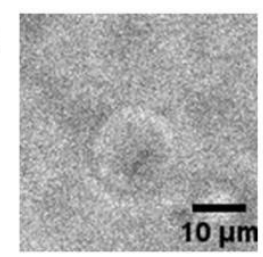

B

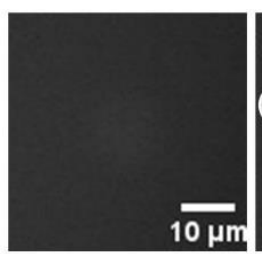

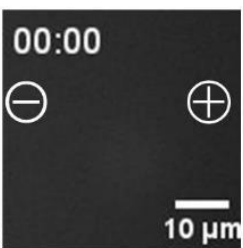

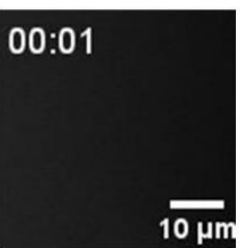

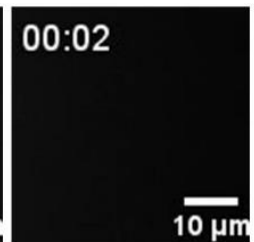

00:06

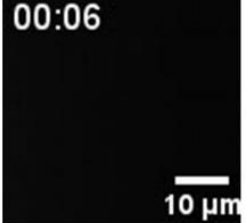

Figure 4. Calcium ion influx was not detected in lecithin/cholesterol = 1:1 (molar ratio) liposomes with $1.1 \mathrm{kV} / \mathrm{cm}, 400 \mu \mathrm{s}$ single PEF. A) Bright-field photo of liposomes before PEF application. B) Fluorescent photos of liposomes with a GFP filter. There was no signal after pulse application. All time displays are in seconds. "00:00" = timing of PEF

480 application. $\oplus=$ the anode side of the electrode; $\ominus=$ the cathode side of the electrode.

481

482

483

484

485

486

487

488

489

490

491

492

493

494

495

496

497

498

499

500

501

502

503

504 
A
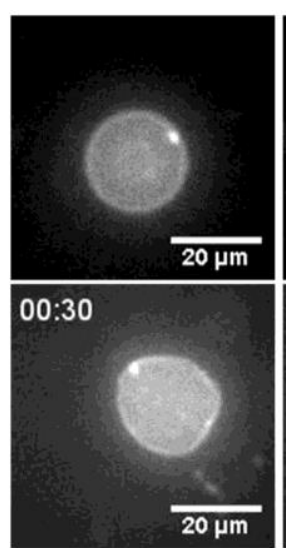

B

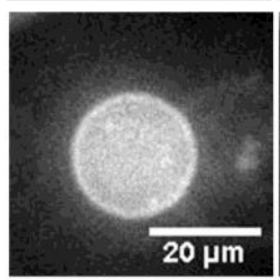

C

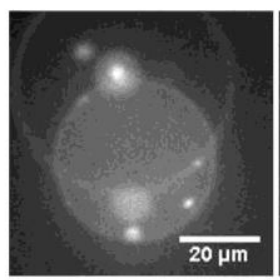

D

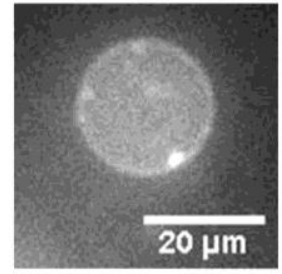

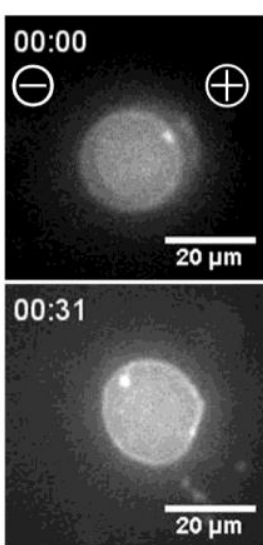
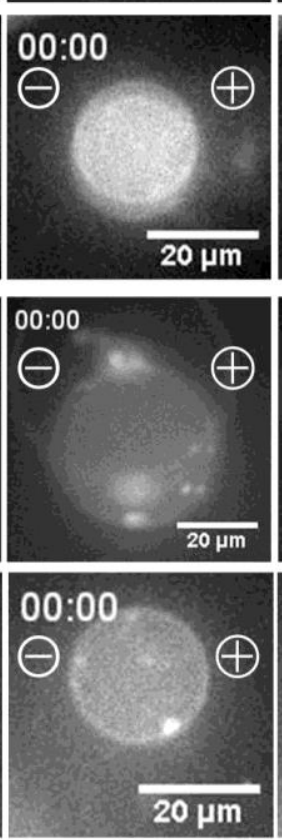
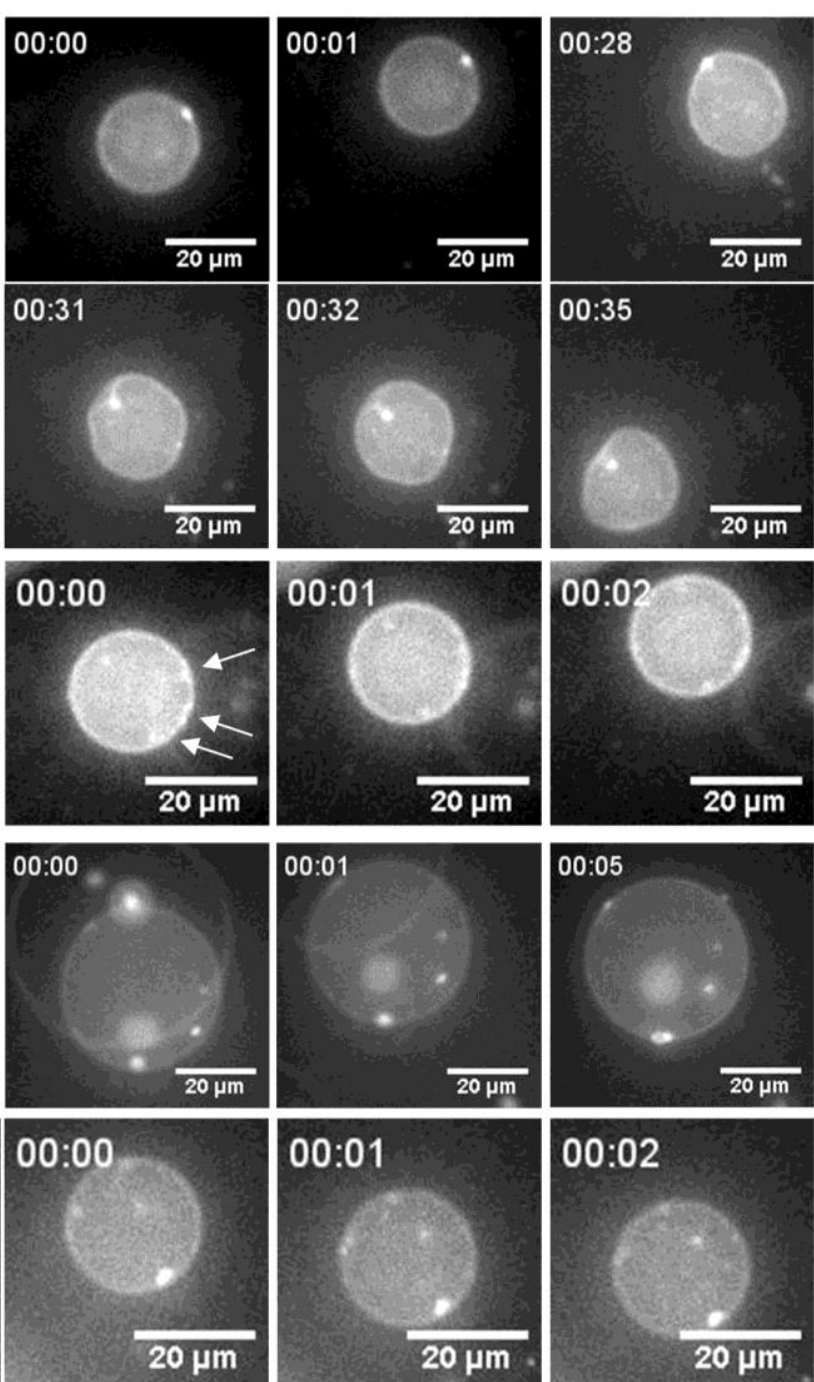
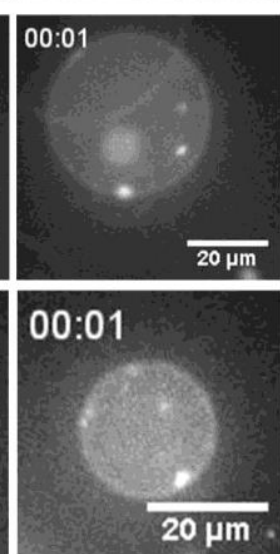
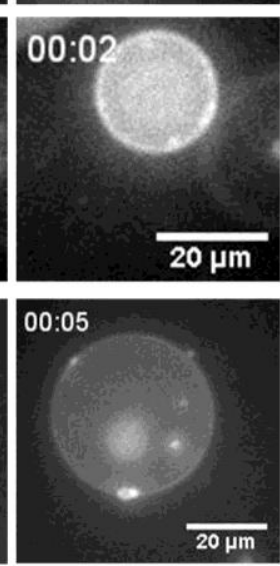

$00: 02$

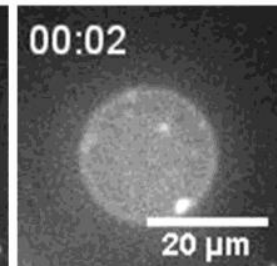

509 Figure 5. (A) Lecithin/cholesterol = 1:1 (molar ratio) liposomes sometimes exhibited

510 endocytosis-like behavior after a single $1.1 \mathrm{kV} / \mathrm{cm}, 400 \mu \mathrm{s}$ PEF application. B)

511 Lecithin/cholesterol = 1:1:1 (molar ratio) liposomes showed patch-like aggregation of

512 lipids on the membrane just after PEF application. White arrows indicate aggregated

513 points. $\mathrm{C}$ and D) DOPC/cholesterol = 1:1 (molar ratio) liposomes did not show patch-like

514 aggregation of lipids on the membrane. All time displays are in seconds. "00:00" =

515 timing of PEF application. $\oplus=$ the anode side of the electrode; $\ominus$ = the cathode side of 516 the electrode. 


\section{Tables}

520 Table 1. Various PEF duration vs. whether the liposome division happened. Electric

521 field was $1.1 \mathrm{kV} / \mathrm{cm}$.

\begin{tabular}{cc}
\hline Pulse duration / us & Division \\
\hline 100 & $\times$ \\
200 & $\times$ \\
300 & $\triangle$ \\
400 & $\circ$ \\
500 & $\circ$
\end{tabular}

$522 \bigcirc=$ liposome division occurred almost every time; $\triangle=$ division occurred some of the 523 time; $\times=$ division did not occur.

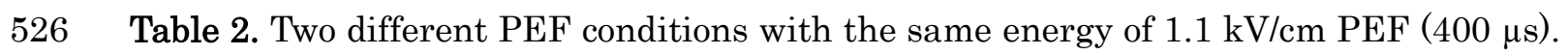

527 Both conditions did not trigger liposome division.

\begin{tabular}{ccc}
\hline Electric field $(\mathrm{kV} / \mathrm{cm})$ & Pulse duration $(\mu \mathrm{s})$ & Division \\
\hline 6.9 & 10 & $\times$ \\
21.9 & 1 & Discharged \\
\hline
\end{tabular}

$\times$ indicates no liposome division

Table 3. Several conductivity patterns of inner and outer liquids. PEF was $1.1 \mathrm{kV} / \mathrm{cm}$ inner liquid.

\begin{tabular}{cc}
\hline Condition & Division \\
\hline$\sigma_{\mathrm{ex}}=1.6 \mathrm{~S} / \mathrm{m}, \sigma_{\text {in }}=0.6 \mathrm{~S} / \mathrm{m}$ & $\circ$ \\
$\sigma_{\mathrm{ex}}=105 \mu \mathrm{S} / \mathrm{m}, \sigma_{\text {in }}=0.6 \mathrm{~S} / \mathrm{m}$ & $\times$ \\
$\sigma_{\mathrm{ex}}=1.6 \mathrm{~S} / \mathrm{m}, \sigma_{\text {in }}=105 \mu \mathrm{S} / \mathrm{m}$ & $\times$ \\
$\sigma_{\mathrm{ex}}=105 \mu \mathrm{S} / \mathrm{m}, \sigma_{\text {in }}=105 \mu \mathrm{S} / \mathrm{m}$ & $\times$ \\
\hline
\end{tabular}

$534 \circ$ indicates liposome division and $\times$ indicates no liposome division. For $\sigma_{\text {in }}=105 \mu \mathrm{S} / \mathrm{m}$,

535 we used sucrose solution and for $\sigma_{\mathrm{ex}}=105 \mu \mathrm{S} / \mathrm{m}$, we used glucose solution. 
A

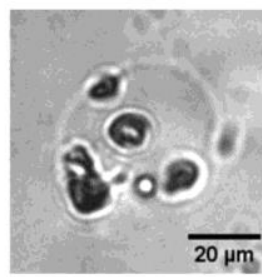

B
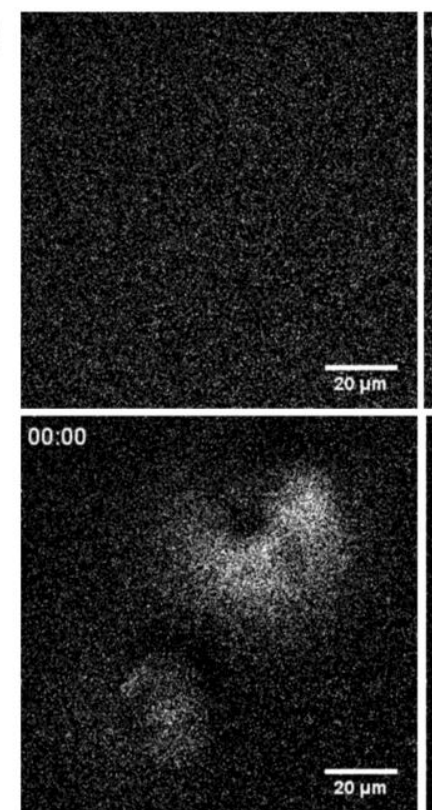

C

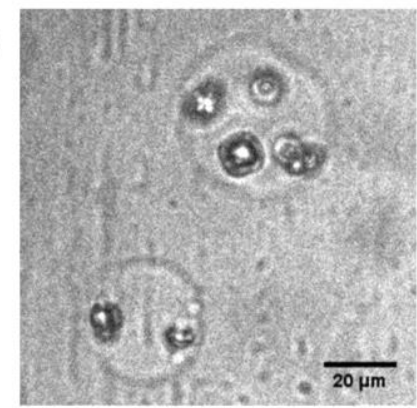

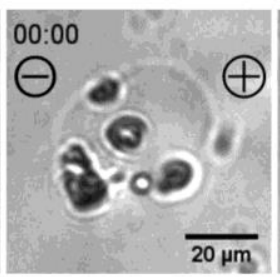
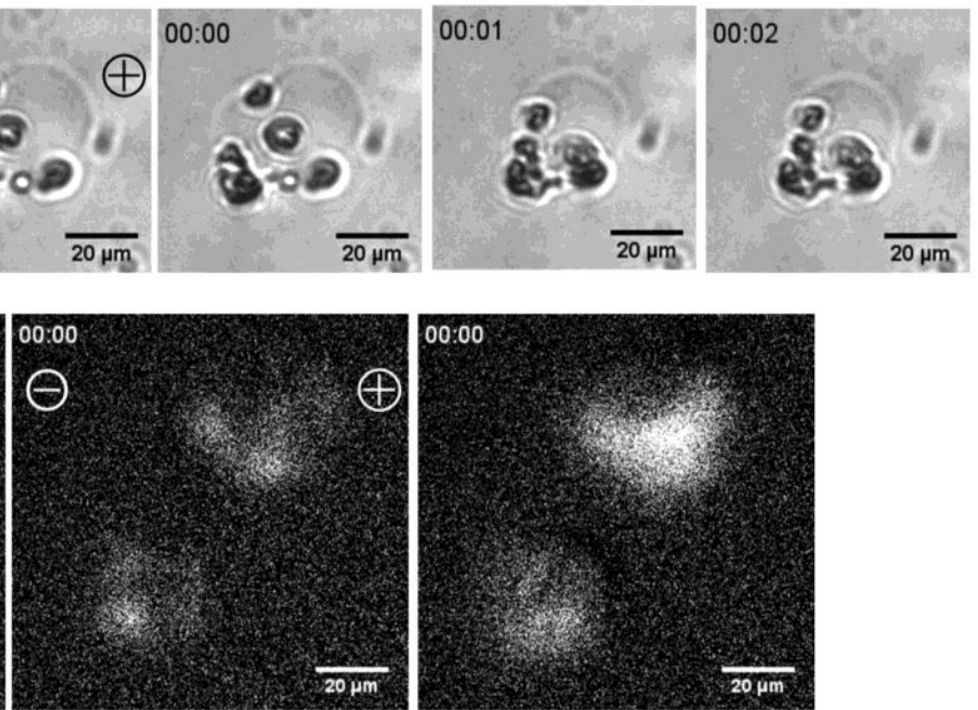

00:01
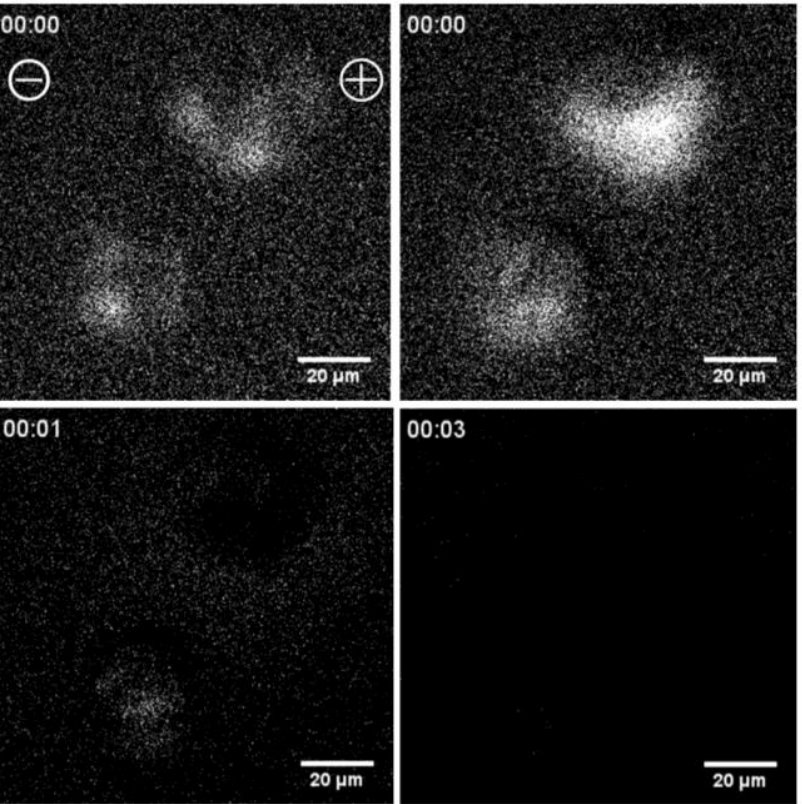

00:03

D

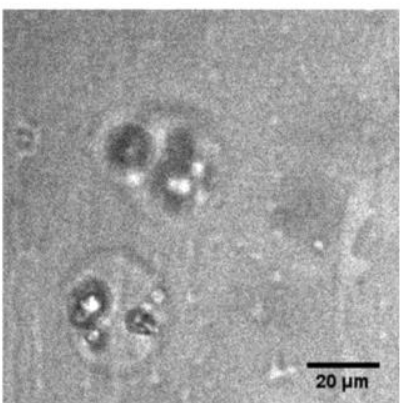

542

543

Supplementary Figure 1. A) Lecithin/cholesterol = 1:1 (molar ratio) liposomes with a

544 rough membrane surface collapsed just after application of a single $1.1 \mathrm{kV} / \mathrm{cm}, 400 \mu \mathrm{s}$

545 PEF. B) Just after integration of lecithin/cholesterol = 1:1 (molar ratio) liposomes, a

546 fluorescent signal emerged within $1 \mathrm{~s}$. C) Bright-field photo of the liposomes before PEF

547 application. D) Bright-field photo of the liposomes after PEF application. All time

548 displays are in seconds. "00:00" = timing of PEF application. $\oplus=$ the anode side of the

549 electrode; $\ominus=$ the cathode side of the electrode. 
A

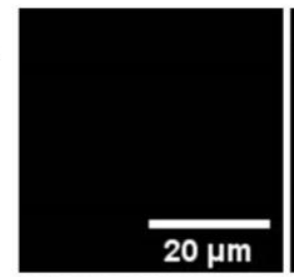

B

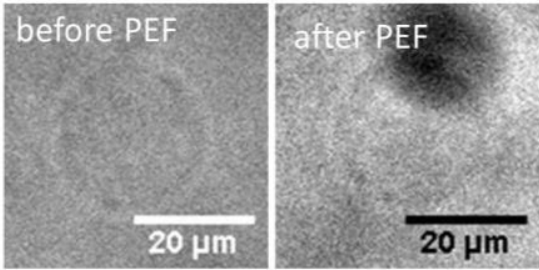

C
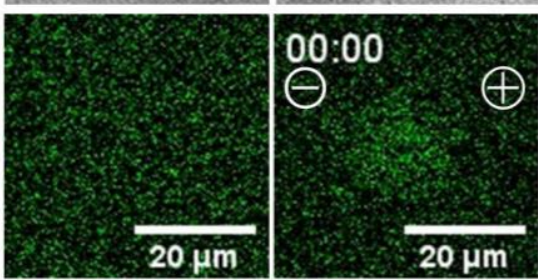

\section{$00: 00$}

D

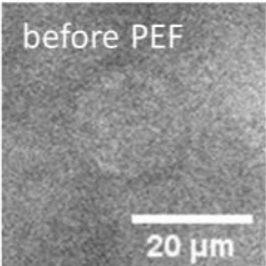

E
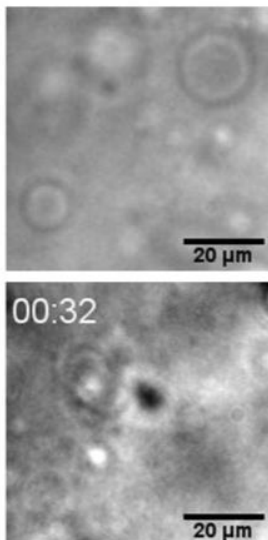

F
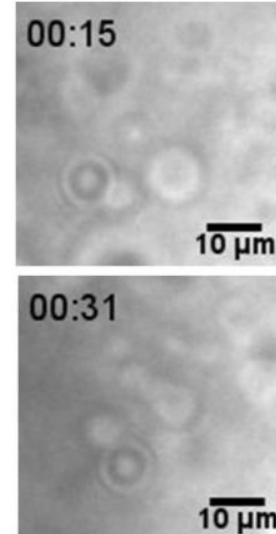

$\overline{10 \mu \mathrm{m}}$

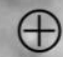

$20 \mu \mathrm{m}$
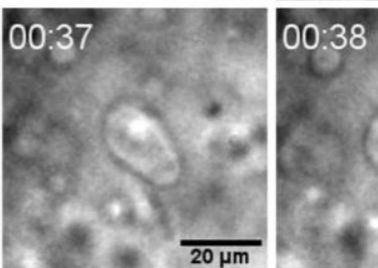

$\overline{20 \mu m}$

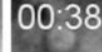

$20 \mu \mathrm{m}$
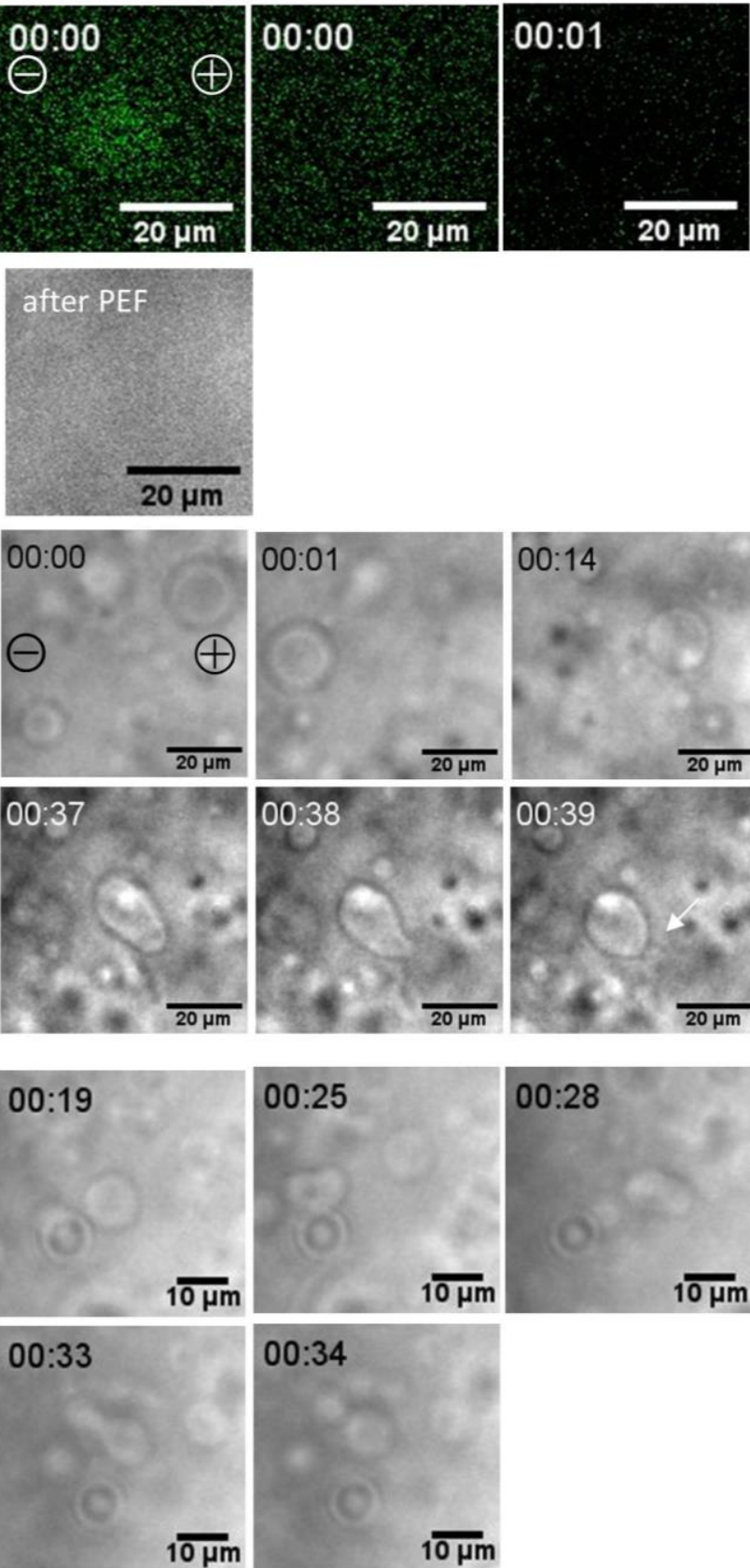

$00: 34$

$\overline{10 \mu \mathrm{m}}$

$\overline{10 \mu \mathrm{m}}$
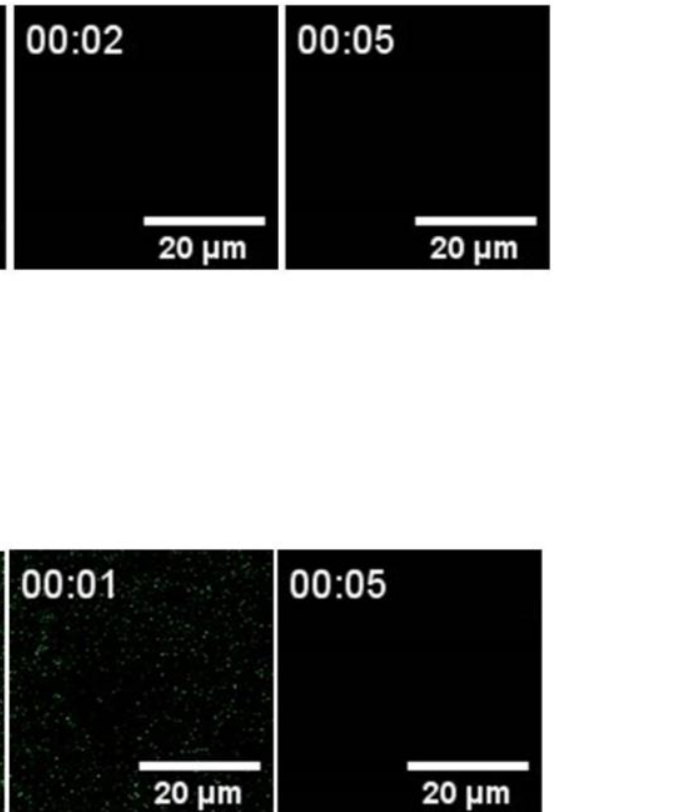

$00: 28$
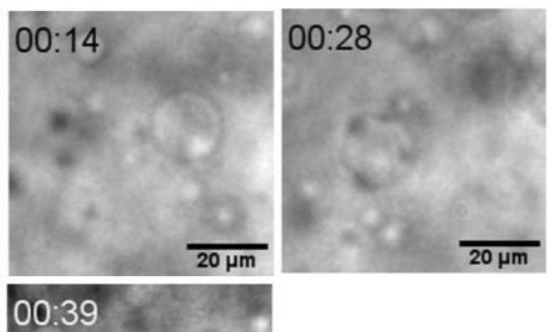

00:39

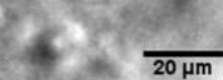

$\overline{10 \mu \mathrm{m}}$ 
551 Supplementary Figure 2. A) Lecithin-only liposomes did not exhibit calcium influx 552 under low-osmotic-pressure outer liquid. B) Lecithin־only liposomes remained after 553 PEF exposure. C) Lecithin/cholesterol = 1:1 (molar ratio) liposomes showed Ca-ion 554 influx just after PEF application under low-osmotic-pressure outer liquid. D) 555 Lecithin/cholesterol = 1:1 (molar ratio) liposomes disappeared after PEF exposure. E) 556 Lecithin/POPG $=2: 1$ (molar ratio) liposomes divided. White arrow indicates the 557 daughter liposome. F) Lecithin/POPC $=2: 1$ (molar ratio) liposomes divided. All time 558 displays are in seconds. "00:00" = timing of PEF application. 